\title{
Bilateral Retinoblastoma Presenting in an in vitro Fertilization Infant with Retinopathy of Prematurity
}

\author{
Ruba Saleh Alghofailia, ${ }^{a}$ Saleh A. Almesfer ${ }^{b}$ \\ aDepartment of Ophthalmology, College of Medicine, Qassim University, Qassim, Saudi \\ Arabia; ${ }^{b}$ Department of Pediatric Ophthalmology, King Khaled Eye Specialist Hospital, \\ Riyadh, Saudi Arabia
}

\section{Keywords}

In vitro fertilization $\cdot$ Leukocoria $\cdot$ Retinopathy of prematurity $\cdot$ Retinoblastoma

\begin{abstract}
Retinopathy of prematurity (ROP) and retinoblastoma (RB) are well-described entities in premature babies. Although their pathogeneses are different, with ROP representing a disorder of interrupted development and RB a genetic disease, a few co-occurring cases have been reported, raising the possibility that the 2 conditions. Here, we report the sixth such case of co-occurring ROP and RB in an 8-month-old infant conceived by in vitro fertilization (IVF) who developed bilateral retinoblastoma a few months after treatment for advanced-stage ROP. While the ROP was initially adequately managed, bilateral RB necessitated bilateral enucleation. This case raises a number of important questions about whether IVF, ROP, and RB are causally related. Although the associations between IVF, ROP, and RB are likely to be coincidental, this case nevertheless highlights that ROP patients require regular follow-up for early diagnosis and treatment of ocular sequelae including RB.
\end{abstract}

\section{Introduction}

Retinopathy of prematurity (ROP) [1] and retinoblastoma (RB) [2] are both well documented in the pediatric population. While the incidence and severity of ROP are inversely related to the infant's weight and gestational age at birth [1], RB has no such associations, being primarily a genetic disease caused by loss of function of the critical retinoblastoma tumor suppressor protein [2]. RB is the most common intraocular tumor; it is highly malignant and, if undetected, can cause significant visual morbidity and mortality [2]. There are 5 
Alghofaili and Almesfer: Bilateral RB Presenting in ROP

previous cases of RB co-occurring with ROP in the literature, either simultaneously or RB occurring months or years after ROP regression [3-6]. Whether ROP predisposes to RB is unknown. Furthermore, in vitro fertilization (IVF) has been suggested to predispose to RB, but the epidemiological data are conflicting [7].

Here, we report a case of RB occurring in an infant conceived by IVF with previous ROP. In addition to providing an opportunity to discuss what is known about the interactions between IVF, ROP, and RB, the case highlights that regular follow-up of ROP patients may help with the early diagnosis and treatment of subsequent visual problems, including RB.

\section{Case Presentation}

A 25 -week gestational age, IVF female infant was born prematurely (weight 650 g). She was hospitalized in the neonatal intensive care unit for 3 months following birth and required supplemental oxygen throughout her admission.

The patient underwent regular ophthalmologic examination during hospitalization. ROP was not apparent at first screening at 27 -weeks gestational age, but by 32 weeks, she had developed severe bilateral stage 3 zone II ROP with severe-plus disease. Unfortunately, there was delay from referring hospital the patient was referred to King Abdulaziz University Hospital (KAUH) at 38 weeks for further evaluation and treatment, by which time she had bilateral ROP: stage 4A on the right and stage 4B zone 1 on the left with plus disease. She was treated with bilateral laser ablation and bevacizumab injection to the left eye before being transferred back to the neonatal intensive care unit.

At 8 months, she was again referred to KAUH due to left-sided orbital cellulitis, which had been treated with intravenous antibiotics in the primary hospital. Clinical examination revealed left-sided proptosis, lid swilling, and conjunctival chemosis. Computed tomography showed multiple calcifications. The patient was referred to King Khaled Specialist Hospital for further evaluation and management of suspected RB. There was no family history of RB. MRI and ultrasound confirmed RB of the left eye and she underwent left-sided enucleation. Histopathological examination confirmed necrotic RB with ciliary body and choroidal invasion. Examination under anesthesia of the right eye showed funnel-shaped retinal detachment but no RB. At follow-up, a second mass was detected in the nasal quadrant of the right eye, which MRI confirmed as RB. She underwent enucleation of the right eye and was referred to pediatric oncology for reevaluation, assessment, and consideration for possible adjuvant chemotherapy. Unfortunately there was no photo before or after treatment.

\section{Discussion/Conclusion}

Here, we describe a preterm infant conceived through IVF with advanced-stage ROP who subsequently developed bilateral RB. This case raises a number of important questions about potential associations between IVF, ROP, and RB.

ROP is a retinal blood vessel development disorder which occurs in low birth weight children [1]. ROP represents a serious and complex disease and is the second leading cause of blindness in the USA behind cortical visual impairment [8]. Prematurity interrupts the development of the retina and retinal vasculature and, as a result, retinal ischemia can occur [9]. Although the pathophysiology of ROP is poorly understood, the compensatory increase in vascular growth factors in response to retinal ischemia results in disordered blood vessel growth [9]. Disease progression can result in vitreous hemorrhage, retinal detachment, and blindness $[1,9]$.

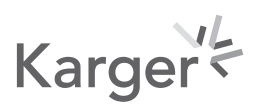


RB is the most common childhood intraocular malignancy, affecting $~ 1: 20,000$ infants with a male:female ratio of $1: 1[10,11]$. RB is caused by inactivating mutations in the retinoblastoma tumor suppressor gene (RB1) $[12,13]$, which, together with subsequent mutations in other genes, results in malignant proliferation and tumor development. RB can be inherited in an autosomal dominant pattern, where 1 mutant allele is inherited through the germ line (35-45\% of cases), and the second allele is sporadically mutated after birth; inherited forms are, therefore, more likely to produce bilateral tumors [13]. However, only $10 \%$ of children with RB have a positive family history, suggesting that the majority of the mutations in heritable RB occur de novo [13].

Our case represents the sixth case of RB occurring in infants with ROP. Vasu et al. [6] reported 2 cases of RB occurring in unrelated children who had previously had ROP; in both cases, the ROP regressed by 38 weeks but the children represented at 4 years with leukocoria. One child was diagnosed with unilateral RB, and the other with bilateral RB. Benz et al. [3] reported a case of simultaneous ROP and bilateral RB occurring in a 40-week gestational age infant with a family history of RB. In their study of 411 very preterm births, Fledelius and Greisen [4] reported a case of one boy with regressed ROP who went on to develop bilateral RB at about 20 months. In the fifth case, similar to our own, Tian et al. [5] reported RB occurring in an IVF infant with ROP.

The incidence of ROP varies by geographical region, and it is estimated that about 32,000 infants become blind or develop severe visual impairment from ROP worldwide each year [14]. Although both RB and ROP are uncommon entities and causality cannot be excluded, with only 5 cases reported in the literature without a family history of RB [4-6], it seems likely that these 2 conditions occurred coincidentally. Furthermore, the pathogeneses of the 2 conditions are entirely different, with one being a genetic disease and the other a consequence of interrupted development $[1,2,10]$, and a unifying mechanism of RB development on a background of ROP is difficult to propose. The cases that have been reported in the literature are also clinically heterogeneous, making it more difficult to draw meaningful conclusions about a causal association between the 2 conditions since there is no obvious temporal pattern of development to suggest a likely pathogenesis. The median age of RB development is 12 months for bilateral tumors [10], so our case is consistent with the usual natural history of RB unmodified by the coexisting ROP.

Perhaps more important than whether the 2 conditions are causally related, our case highlights that clinicians must remain vigilant for coexisting eye disease in infants with ROP during regular follow-up visits to detect other ocular associations with ROP including refractive errors, strabismus, and glaucoma [1]. It is also worth noting that leukocoria (white reflex, or white pupil, instead of the normal red reflex) is a common presenting feature of both ROP and RB, so vigilance for RB must be maintained in children with leukocoria due to ROP [15].

Our case represents the second case of RB occurring with ROP in an infant conceived by IVF [5], raising the possibility that IVF may predispose to RB; indeed, IVF has been suggested to cause epigenetic abnormalities in offspring that predispose to various diseases $[7,16]$. Although the literature is sparse, there is some evidence to suggest that IVF increases the risk of RB $[17,18]$, although several studies have failed to find this association, including in large population-based studies $[19,20]$. Again, given the weak large-scale epidemiological data, it seems more likely that the 2 conditions occurred coincidentally.

The co-occurrence of ROP and RB in an IVF infant, although rare and possibly coincidental, highlights that premature infants may develop other eye problems. Awareness among parents and clinicians of the possible occurrence of ocular abnormalities other than ROP in premature babies, facilitated by regular screening and patient education for ROP, could lead to parents reporting abnormalities to the treating ophthalmologist early. Children with ROP

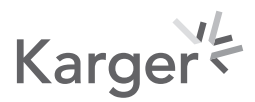


should receive regular follow-up to detect and treat other ocular problems. Further reports of associations between IVF, ROP, and RB might shed further light on any possible causal mechanisms.

\section{Statement of Ethics}

This study was conducted in accordance with the ethical requirements of the Declaration of Helsinki; written informed consent for publication of her case was obtained from the patient's parents.

\section{Conflict of Interest Statement}

The authors declare no conflicts of interest.

\section{Funding Sources}

The authors did not receive any funding.

\section{Author Contributions}

All the authors attest that they meet the current ICMJE criteria for authorship. Ruba alghofaili: data acquisition, literature search, manuscript preparation, and manuscript editing. Saleh Almesfer followed up the patient, performed the surgery, and manuscript review. All the authors approved the final version of the manuscript and are accountable for all aspects of the work. All the authors attest that they meet the current ICMJE criteria for authorship.

\section{References}

1 Bashinsky AL. Retinopathy of prematurity. N C Med J. 2017;78(2):124-8.

2 Dimaras H, Kimani K, Dimba EA, Gronsdahl P, White A, Chan HS, et al. Retinoblastoma. Lancet. 2012;379(9824): 1436-46.

3 Benz MS, Escalona-Caamaño EM, Murray TG. Simultaneous presentation of retinopathy of prematurity and bilateral familial retinoblastoma in a premature infant. J Pediatr Ophthalmol Strabismus. 2003;40(2):98-100.

4 Fledelius HC, Greisen G. Very pre-term birth and visual impairment. A retrospective investigation of 411 infants of gestational age 30 weeks or less, 1983-89 Rigshospitalet, Copenhagen. Acta Ophthalmol Suppl. 1993;(210):63-5.

5 Tian T, Ji XD, Zhang Q, Peng J, Zhao PQ. A delayed diagnosis of unsuspected retinoblastoma in an in vitro fertilisation infant with retinopathy of prematurity. Int J Ophthalmol. 2016;9(9):1361-3.

6 Vasu U, Nithyanandam S, D'Souza S, Kamath S. Retinoblastoma in patients with regressed retinopathy of prematurity. Indian J Ophthalmol. 2011;59(6):501-2.

7 Foix-L'Helias L, Aerts I, Marchand L, Lumbroso-Le Rouic L, Gauthier-Villars M, Labrune P, et al. Are children born after infertility treatment at increased risk of retinoblastoma? Hum Reprod. 2012;27(7):2186-92.

8 Sommer A, Taylor HR, Ravilla TD, West S, Lietman TM, Keenan JD, et al. Challenges of ophthalmic care in the developing world. JAMA Ophthalmol. 2014;132(5):640-4.

9 Hartnett ME, Penn JS. Mechanisms and management of retinopathy of prematurity. N Engl J Med. 2013; 368(12):1162-3.

10 Melamud A, Palekar R, Singh A. Retinoblastoma. Am Fam Physician. 2006;73(6):1039-44.

11 Kivela T. The epidemiological challenge of the most frequent eye cancer: retinoblastoma, an issue of birth and death. Br J Ophthalmol. 2009;93(9):1129-31.

12 Friend SH, Bernards R, Rogelj S, Weinberg RA, Rapaport JM, Albert DM, et al. A human DNA segment with properties of the gene that predisposes to retinoblastoma and osteosarcoma. Nature. 1986;323(6089):643-6. 
13 Mallipatna A, Marino M, Singh AD. Genetics of retinoblastoma. Asia Pac J Ophthalmol. 2016;5(4):260-4.

14 Blencowe H, Lawn JE, Vazquez T, Fielder A, Gilbert C. Preterm-associated visual impairment and estimates of retinopathy of prematurity at regional and global levels for 2010. Pediatr Res. 2013;74(Suppl 1):35-49.

15 Balmer A, Munier F. Differential diagnosis of leukocoria and strabismus, first presenting signs of retinoblastoma. Clin Ophthalmol. 2007;1(4):431-9.

16 DeAngelis AM, Martini AE, Owen CM. Assisted reproductive technology and epigenetics. Semin Reprod Med. 2018;36(3-4):221-32

17 Marees T, Dommering CJ, Imhof SM, Kors WA, Ringens PJ, van Leeuwen FE, et al. Incidence of retinoblastoma in Dutch children conceived by IVF: an expanded study. Hum Reprod. 2009;24(12):3220-4.

18 Moll AC, Imhof SM, Cruysberg JR, Schouten-van Meeteren AY, Boers M, van Leeuwen FE. Incidence of retinoblastoma in children born after in-vitro fertilisation. Lancet. 2003;361(9354):309-10.

19 Bradbury BD, Jick H. In vitro fertilization and childhood retinoblastoma. Br J Clin Pharmacol. 2004;58(2): 209-11.

20 Lidegaard O, Pinborg A, Andersen AN. Imprinting diseases and IVF: Danish National IVF cohort study. Hum Reprod. 2005;20(4):950-4. 